\title{
ON LACUNARY SETS FOR NONABELIAN GROUPS
}

\author{
A. H. DOOLEY \\ (Received 8 September 1976; revised 1 July 1977) \\ Communicated by E. Strzelecki
}

\begin{abstract}
Results concerning a class of lacunary sets are generalized from compact abelian to compact nonabelian groups. This class was introduced for compact abelian groups by Bozejko and Pytlik; it includes the $p$-Sidon sets of Edwards and Ross. A notion of test family is introduced and is used to give necessary conditions for a set to be lacunary. Using this, it is shown that $\mathscr{S} \mathscr{U}(2)$ has no infinite $p$-Sidon sets for $1 \leqslant p<2$.
\end{abstract}

Subject classification (Amer. Math. Soc. (MOS) 1970): 42 A 44, 43 A 14.

\section{Introduction}

In the recent literature there has been considerable interest in the existence of certain types of lacunary or "thin" subsets of the dual objects of compact nonabelian groups, since in the abelian case, these have proved to be a useful tool. But the results have been disappointing; many well-behaved non-abelian groups, even compact connected Lie groups, can fail to have infinite central Sidon sets in their dual object (Price, 1971; Rider, 1972).

Bozejko and Pytlik (1972), in the case of an abelian group, provided a unifying concept for lacunary sets (their sets of type $S_{p, q}$ and $S_{p, q}^{*}$ include most types of lacunary sets previously considered) and at the same time introduced some $a$ priori new families of lacunary sets. Edwards and Ross (1974) show that some of their families are strictly larger than previously known families of lacunary sets, but only for the abelian case. So it is natural to enquire whether their ideas give any interesting results in the non-abelian case.

This article is the first of a series in which I shall consider this question. In later articles I shall be principally interested in results for compact connected Lie groups-here I shall deal mostly with the case of an arbitrary compact group.

Closer examination of Bozejko and Pytlik (1972) shows that many of the results therein (in particular Theorems 1 and 2) have straightforward generalizations to the non-abelian case-I summarize these in Section 3. Section 4 is devoted to central and local central analogues of these sets (cf. Parker (1972)). In Section 5, I consider $q$-Sidon and central $q$-Sidon sets, giving a generalization of the theorem that a Sidon set is $\Lambda(p)$ for all $p>1$. Section 6 contains some 
necessary conditions for a set to be of type $\Lambda(p, q)$ in terms of the hypergroup structure on the dual. In particular, the notion of a test family is extended from the abelian case. This yields an improvement to a theorem of Benke (1974), and as an application, it is shown that $\mathscr{S} \mathscr{U}(2)$ has no infinite $p$-Sidon sets for $1 \leqslant p<2$.

I remark here that there are many interesting questions left unanswered by this article. For example, the method of Cecchini (1972) for showing that the dual objects of compact Lie groups admit no infinite local central $\Lambda(4)$ sets has no extension to sets of type $\Lambda(p, q)$, and other methods have to be developed. Rider's proof (1972) that compact connected semi-simple Lie groups have no infinite central Sidon sets appears to admit no generalization to central $p$-Sidon sets $(1<p<2)$. I shall deal with these questions in future articles.

\section{Notation}

$\mathbf{R}$ denotes the set of real numbers. If $p \in[1, \infty], p^{\prime} \in[1, \infty]$ is such that $(1 / p)+\left(1 / p^{\prime}\right)=1$. Unless specified otherwise, $G$ will be a compact Hausdorff group with Haar measure $\lambda_{G^{*}}$. The symbol $L^{p}(G)(1 \leqslant p \leqslant \infty)$ will denote the usual $L^{p}$-spaces, $C(G)$ the continuous functions, $T(G)$ the trigonometric polynomials on $G, M(G)$ the space of Radon measures on $G$. If $A \subseteq M(G), Z A$ will denote the convolution centre of $A$. The dual object of $G$, denoted $\Sigma$, is a maximal set of pairwise inequivalent irreducible representations. For $\sigma \in \Sigma$, let $d_{\sigma}$ denote the degree of $\sigma, \chi_{\sigma}$ the character of $\sigma$. If $\sigma, \eta \in \Sigma$ we may write $\chi_{\sigma} \chi_{\eta}=\sum_{\zeta \in \Sigma} n_{\sigma \eta}(\zeta) \cdot \chi_{\zeta}$, corresponding to the decomposition of the tensor product $\sigma \otimes \eta$, where $n_{\sigma \eta}(\zeta)$ is a positive integer. The (finite) support of $n_{\sigma \eta}$ is written $\sigma \times \eta$. For any $R \subseteq \Sigma$, let $\mathscr{E}^{p}(R)(1 \leqslant p \leqslant \infty)$ be the spaces introduced in Section 28 of Hewitt and Ross (1972). $\mathscr{Z} \mathscr{E}^{p}(R)$ is the set of elements of $\mathscr{E}^{p}(R)$ which commute with every element of $\mathscr{E}^{p}(R)$ under multiplication. Note that $\mathscr{Z}_{\mathscr{E}} p(R)=\left\{A \in \mathscr{E}^{p}(R) \mid\right.$ for $\sigma \in R, A_{\sigma}$ is a multiple of the identity\}; the map $\left\langle a_{\sigma} I_{a_{\sigma}}\right\rangle \mapsto d_{\sigma}^{2 / p} . a_{\sigma}$ is an isometric isomorphism of $\mathscr{Z} \mathscr{E} p(R)$ onto $l^{p}(R)$. For $\mu \in M(G), \hat{\mu} \in \mathscr{E}^{\infty}(\Sigma)$, defined by $\hat{\mu}(\sigma)=\int_{G} \bar{\sigma}(x) d \mu(x)$, is its Fourier transform. If $A \subseteq M(G), R \subseteq \Sigma, A_{R}=\{\mu \in A \mid \hat{\mu}(\sigma)=0$ for $\sigma \notin R\}$.

Further clarification of these concepts may be found in Hewitt and Ross (1972), as may any unexplained notation or terminology.

3. Definitions and elementary properties; sets of type $V(p, q)$ and $\Lambda(p, q)$

(3.1) Defintion. Let $G$ be a compact group, $R \subseteq \Sigma$, and suppose $p, q \in[1, \infty]$. Then $R$ is of type $V(p, q)$ if

(i) there exists $\kappa \in \mathbf{R}$ such that for all $f \in T_{R}(G)$,

$R$ is of type $\Lambda(p, q)$ if

$$
\|\hat{f}\|_{\alpha^{\prime}} \leqslant \kappa\|f\|_{p^{\prime}}
$$

(ii) there exists $\kappa \in \mathbf{R}$ such that for all $f \in T_{R}(G)$,

$$
\|f\|_{p} \leqslant \kappa\|\hat{f}\|_{q} \text {. }
$$


For typographical and historical reasons, I have preferred to alter the notation of Bozejko and Pytlik (1972). The correspondence is given by

$$
\begin{array}{lll}
R \text { is of type } S_{p, q} & \text { if and only if } & R \text { is of type } V\left(p^{\prime}, q^{\prime}\right) ; \\
R \text { is of type } S_{p, q}^{*} & \text { if and only if } & R \text { is of type } \Lambda\left(p^{\prime}, q^{\prime}\right) .
\end{array}
$$

Notice that for $p>2, R$ is of type $\Lambda(p)$ if and only if $R$ is of type $\Lambda(p, 2)$, and also that $R$ is of type $\Lambda(2)$ if and only if there exists $p>2$ such that $R$ is of type $V(p, 2)$. Every set of type $\Lambda(p)(p<2)$ is also of type $V(\infty, p)$.

The proofs of the following theorems may safely be left to the reader familiar with Bozejko and Pytlik (1972), Edwards and Ross (1974) and Section 37 of Hewitt and Ross (1972).

(3.2) THEOREM. Let $G$ be a compact group, $p, q \in[1, \infty], q \neq 1$, and suppose that $R \subseteq \Sigma$. The following propositions are pairwise equivalent:

(i) $R$ is of type $V(p, q)$.

(ii) There is $\kappa \in \mathbf{R}$ such that for all $f \in C_{R}(G)$ (or $L_{R}^{p^{\prime}}(G)$ ), $\hat{f} \in \mathscr{E}^{q^{\prime}}(\Sigma)$ and $\|\hat{f}\|_{q^{\prime}} \leqslant \kappa\|f\|_{p^{\prime}}$

(iii) $\left(L_{R}^{p^{\prime}}\right)^{\wedge} \subseteq \mathscr{E}^{q^{\prime}}(\Sigma)$.

(iv) $\left.\left(L^{p}\right)^{\wedge}\right|_{R} \supseteq \mathscr{E} q(R)\left(\right.$ or $\mathscr{E}_{0}(R)$ if $p=1$ and $\left.q=\infty\right)$.

(3.3) THEOREM. Let $G$ be a compact group, $p, q \in[1, \infty], p \neq \infty$, and suppose that $R \subseteq \Sigma$. The following statements are pairwise equivalent:

(i) $R$ is of type $\Lambda(p, q)$.

(ii) There is $\kappa \in \mathbf{R}$ such that for all $f \in C_{R}(G)$ (or $L_{R}^{\infty}(G)$ ), $\hat{f} \in \mathscr{E}^{q}(\Sigma)$ implies that $\|f\|_{p} \leqslant\|\hat{f}\|_{q}$.

(iii) (only for $p \neq 1$ ). There is $\kappa \in \mathbf{R}$ such that for all $\nu \in \mathbf{M}(G), \hat{v} \in \mathscr{E}^{q}(R)\left(\right.$ or $\mathscr{E}_{0}(R)$ if $q=\infty)$ implies that $\nu \in L^{p}(G)$ and $\|\nu\|_{p} \leqslant \kappa\|\hat{\nu}\|_{q}$.

(iv) $\left.\left(L_{R}^{p}\right)^{\wedge}\right|_{R} \supseteq \mathscr{E}^{q}(R)$.

(v) $\left.\left(L^{p^{\prime}}\right)^{\wedge}\right|_{R} \supseteq \mathscr{E}^{\mathscr{q}}(R)\left(\right.$ if $\left.p=1,\left.C(G)^{\wedge}\right|_{R} \subseteq \mathscr{E}^{q^{\prime}}(R)\right)$.

If $p=\infty$, one has that (i) implies (iv) and that (iv) and (v) are equivalent.

Various other equivalent statements are available in certain cases (for example, for $p=1$ or $\infty$ in (3.2)).

(3.4) Remarks. The above theorems show that every set of type $\Lambda(p, q)$ is also of type $V(p, q)$. It is clear also that each type is hereditary, that is, a subset of a set of type $V(p, q)$ or $\Lambda(p, q)$ is of the same type. Furthermore, it is easily seen that if $p_{1} \leqslant p, q_{1} \leqslant q$ then every set of type $V(p, q)$ (respectively $\Lambda(p, q)$ ) is also of type $V\left(p_{1}, q_{1}\right)$ (respectively $\Lambda\left(p_{1}, q_{1}\right)$ ).

Of course, for many values of $(p, q)$ the class of sets of type $V(p, q)$ is trivial; either it includes all subsets of $\Sigma$ or it includes only finite sets (which are clearly of 
type $V(p, q)$ for all $(p, q))$. A consequence of the Hausdorff-Young inequality is that every subset of $\Sigma$ is of type $\Lambda\left(p, p^{\prime}\right)$ for all $2 \leqslant p<\infty$. Also note here that the preceding theorems show $\Sigma$ to be of type $V(p, q)$ if and only if $\Sigma$ is of type $\Lambda(p, q)$.

\section{Central and local central sets}

(4.1) Definition. Following Parker (1972), I shall say that $R \subseteq \Sigma$ is of type central $V(p, q)$ if (3.1) (i) holds for all $f \in Z T_{R}(G) . R$ is of type local central $V(p, q)$ if (3.1) (i) holds as $f$ runs through all irreducible characters of $G$. In a similar way, one defines sets of type central $\Lambda(p, q)$ and local central $\Lambda(p, q)$.

(4.2) Remarks. Analogues of Theorems (3.2) and (3.3) hold for sets of type central $V(p, q)$ and central $\Lambda(p, q)$ (one replaces, in the statements, $T(G), C(G)$, $L^{p}(G), M(G)$ and $\mathscr{E}^{p}(\Sigma)$ by $Z T(G), Z C(G), Z L^{p}(G), \mathbf{Z M}(G)$ and $\left.\mathscr{Z} \mathscr{E}^{p}(G)\right)$. Again, the proofs are similar to those of Bozejko and Pytlik (1972), using Lemma 1.1 of Parker (1972) where necessary. It is clear that $R$ is of type $V(p, q)$ implies that $R$ is of type central $V(p, q)$ and this implies that $R$ is of type local central $V(p, q)$, and similarly for type $\Lambda(p, q)$.

(4.3) Lemma. Let $\sigma \in \Sigma$.

(i) If $q \in[1, \infty],\left\|\hat{\chi}_{\sigma}\right\|_{q}=d_{\sigma}^{1-2 / q}$.

(ii) If $p \leqslant 2, d_{\sigma}^{1-2 / p} \leqslant\left\|\chi_{\sigma}\right\|_{p}$, and if $p \geqslant 2,\left\|\chi_{\sigma}\right\|_{p} \leqslant d_{\sigma}^{1-2 / p}$.

Proor. Let $\tau \in \Sigma$. Then

$$
\hat{\chi}_{\sigma}(\tau)= \begin{cases}\frac{1}{d_{\sigma}} I_{d_{\sigma}} & \text { if } \sigma=\tau \\ 0 & \text { if } \sigma \neq \tau .\end{cases}
$$

Hence $\|\hat{\chi}\|_{q}=d_{\sigma}^{(2-q) / q}=d_{\sigma}^{1-2 / q^{\prime}}$. The statement (ii) follows from (i) by the Hausdorff-Young inequality and its converse.

(4.4) Proposition. (i) $\Sigma$ is of type local central $V(1, q)$ for all $q \in[1, \infty]$.

(ii) Suppose that $p \in] 1,2], q \in] 2, \infty]$ and $p>q^{\prime}$. Then if $R \subseteq \Sigma$ is of type local central $V(p, q), \sup \left\{d_{\sigma} \mid \sigma \in R\right\}<\infty$. Further, if $R$ is of type central $V(p, q), R$ is finite.

(iii) Suppose that $q \in[1, \infty]$. Then if $R \subseteq \Sigma$ is of type local central $\Lambda(\infty, q)$, $\sup \left\{d_{\sigma} \mid \sigma \in R\right\}<\infty$. If $R$ is of type central $\Lambda(\infty, q), R$ is finite.

Proof. By (4.3) (i), sup $\left\{\left\|\hat{\chi}_{\sigma}\right\|_{q^{\prime}} /\left\|\chi_{\sigma}\right\|_{\infty} \mid \sigma \in \Sigma\right\} \leqslant \sup \left\{d_{\sigma}^{-2 / q} \mid \sigma \in \Sigma\right\} \leqslant 1$. This proves (i). To show (ii), first notice that if $R$ is of type local central $V(p, q)$ for $p \leqslant 2$, then $\sup \left\{\left\|\hat{\chi}_{\sigma}\right\|_{q^{\prime}} /\left\|\chi_{\sigma}\right\|_{p^{\prime}} \mid \sigma \in R\right\}<\infty$. By (4.3) (ii), this implies that

$$
\sup \left\{d_{\sigma}^{\left(2 / p^{\prime}\right)-(2 / q)} \mid \sigma \in R\right\}<\infty
$$

and so either $p \leqslant q^{\prime}$ or $\sup \left\{d_{\sigma} \mid \sigma \in R\right\}<\infty$. 
Suppose now that $R$ is in infinite set of type central $\Lambda(p, q)\left(p \geqslant 2, p>q^{\prime}\right)$. Then $\sup \left\{d_{\sigma} \mid \sigma \in R\right\}<\infty$, so by Theorem 2.4 and Corollary 3.4 of Parker (1972), we may assume that $R$ is of type central $\Lambda\left(p^{\prime}\right)$. Then $Z E^{q}(R) \supseteq\left(Z L_{R}^{p}\right)^{\wedge}=\left(Z L_{R}^{2}\right)^{\wedge}=Z \mathscr{E}^{2}(R)$. This implies that $R$ is finite or that $q \leqslant 2$. The latter is impossible.

(iii) is similar, except that one uses the fact that any infinite set of uniformly bounded degree contains an infinite central Sidon set (see Parker (1972), Corollary 3.4).

(4.5) Remark. Suppose that $q \in] 2, \infty], p \in] 1,2]$, and suppose that $R$ is an infinite set of type central $V(p, q)$. Then the argument of (ii) shows that $R$ contains no infinite set of type central $\Lambda\left(p^{\prime}\right)$. If $G$ is abelian, every infinite set contains such a subset. However, in the nonabelian case, not every infinite set need contain an infinite central $\Lambda\left(p^{\prime}\right)$ set (Cecchini (1972), Theorem 3; Rider (1972), Example 1).

\section{Some results on $q$-Sidon sets}

Sets of type (central) $V\left(1, q^{\prime}\right)$ are said to be (central) $q$-Sidon sets. These sets were discussed for $G$ abelian by Edwards and Ross (1974) and L.-S. Hahn. By the Hausdorff-Young inequality, $\Sigma$ is $q$-Sidon for all $q \geqslant 2$. Hence I shall consider only $1 \leqslant q<2$. (Central) 1 -Sidon sets are (central) Sidon sets.

(5.1) TheOREM. Let $R \subseteq \Sigma$ be $q$-Sidon. Then

(i) $R$ is of type $V(\infty, 2 q /(3 q-2))$.

(ii) $R$ is of type $\Lambda(r, 2 q /(3 q-2))$ for all $r \in[1, \infty[$; in fact there is $\kappa \in \mathbf{R}$ such that for all $f \in T_{R}(G)$ and for every $r \in\left[1, \infty\left[,\|f\|_{r} \leqslant \kappa r^{\frac{1}{k}}\|\hat{f}\|_{2 q /(3 q-2)}\right.\right.$.

Proof. (i) is proved as in Theorem 2.1 of Edwards and Ross (1974). The proof relies on the fact that $\mathscr{M}(C(G), \mathscr{E} q(\Sigma))=\mathscr{E}^{2 q /(2-q)}(\Sigma)$.

A version of (ii) for abelian groups is proved by Bozejko and Pytlik (1972), Theorem 2. Using methods of Figà-Talamanca and Rider (1966), I shall indicate how to extend this proof to the non-abelian case.

Let $\mathscr{G}=\Pi_{\sigma \in R} \mathscr{U}\left(d_{\sigma}\right)$; let $f \in T_{R}(G)$, and $0<\alpha<1$. Define

$$
f_{\alpha}: G \times \mathscr{G} \rightarrow \mathbf{C}:(x, W) \mapsto \sum_{\sigma \in R} d_{\sigma} \operatorname{Tr}\left[\hat{f}(\sigma) \cdot|\hat{f}(\sigma)|^{-\alpha} \cdot W_{\sigma} \cdot \sigma(x)\right] .
$$

Note that for $W \in \mathscr{G}, \sigma \mapsto W_{\sigma}^{*}|\hat{f}(\sigma)|^{\alpha} \in \mathscr{E}_{00}(\Sigma)$. Since $R$ is $q$-Sidon, there exists $\kappa \in \mathbf{R}$ (independent of $f$ ) and there exists $g_{W} \in L^{1}(G)$ (depending on $f$ ) such that

$$
\hat{g}_{W}(\sigma)=W_{\sigma}^{*}|\hat{f}(\sigma)|^{\alpha} \text { and }\left\|g_{W}\right\|_{1} \leqslant \kappa\left\|\left.W^{*}|\hat{f}|_{R}\right|^{\alpha}\right\|_{q^{\prime}} \text {. }
$$

It is easy to see that $\left[\left(f_{\alpha}(\cdot, W)\right)^{\wedge} \cdot \hat{\mathrm{g}}_{W}\right](\zeta)=\hat{f}(\zeta)$ for all $\zeta \in \Sigma$, so that

Hence, for $1 \leqslant r<\infty$,

$$
f=f_{\alpha}(\cdot, W) * g_{W} \cdot
$$

$$
\|f\|_{r}^{r} \leqslant\left\|f_{\alpha}(\cdot, W)\right\| r\left\|_{r} g_{W}\right\|_{1}^{r}
$$


If $\alpha \geqslant 1 / q$, (D.41) and (D.48) of Hewitt and Ross (1972) show that for $\sigma \in R$, $\left\|W_{\sigma}|\hat{f}(\sigma)|^{\alpha}\right\|_{\phi_{q^{\prime}}^{\prime}}^{q^{\prime}}=\|\hat{f}(\sigma)\|_{\hat{\phi}_{\alpha q}}^{\alpha q^{\prime}}$ and so (1) implies that

$$
\left\|g_{W}\right\|_{1} \leqslant \kappa\|\hat{f}\|_{\alpha q^{\prime}}^{\alpha}
$$

Combining (2) and (3) and integrating over $\mathscr{G}$, one obtains

$$
\|f\|_{r}^{r} \leqslant \kappa\|\hat{f}\|_{\alpha q^{\prime}}^{\alpha r} \int_{G} \int_{\mathscr{G}}\left|f_{\alpha}(x, W)\right|^{r} d W d x
$$

Since $R$, considered as a subset of the dual $\Sigma(\mathscr{G})$ of $\mathscr{G}$, is a Sidon set (Hewitt and Ross, 1972 (36.1)), there exists $B \in R$, independent of $f$, such that for all $r \in[2, \infty)$ and for each $x \in G$,

$$
\begin{aligned}
\int_{g}\left|f_{\alpha}(x, W)\right|^{r} d W & =\left\|f_{\alpha}(x, \cdot)\right\| \|_{r} \leqslant\left(B \sqrt{ }\left\|f_{\alpha}(x, \cdot)\right\|_{2}\right)^{r} \\
& \leqslant\left(B \sqrt{ }\|\hat{f}\|_{2(1-\alpha)}^{(1-\alpha)}\right)^{r},
\end{aligned}
$$

provided that $1-\alpha \geqslant \frac{1}{2}$. Now (4) and (5) give

$$
\left.\|f\|_{r} \leqslant B \kappa \sqrt{ }\|\hat{f}\|\right)_{2(1-\alpha)}^{(1-\alpha)}\|\hat{f}\|_{\alpha \alpha^{\prime}}^{\alpha},
$$

provided that $(1-\alpha) \geqslant \frac{1}{2}$ and $\alpha q^{\prime} \geqslant 1$. Choosing $\alpha$ such that $\alpha q^{\prime}=2(1-\alpha)$, it is easily established that $\alpha q^{\prime}=2 q /(3 q-2)$, which completes the proof.

(5.2) REMARK. I do not know whether (5.1) (i) has any analogue for central $q$-Sidon sets (even those with uniformly bounded degree). I can show that a central $q$-Sidon set of uniformly bounded degree satisfies (5.1) (ii) with " $f \in T_{R}(G)$ " replaced by " $f \in Z T_{R}(G)$ ". Parker (1972) has shown that a central Sidon set of uniformly bounded degree is actually $\Lambda(p)$ for all $p$.

\section{Test families for non-abelian groups}

(6.1) Definition. For $R \subseteq \Sigma$, let $\nu(R)=\Sigma_{\sigma \in R} d_{\sigma}^{2}$. If $G$ is abelian, $\nu(R)$ is simply the cardinality of $R$.

(6.2) Lemma. Let $P, Q$ be non-empty finite subsets of $\Sigma$. There exists $k \in Z T(G)$ such that

(i) $\hat{k}(\sigma)=I_{d_{\sigma}}, \sigma \in P$;

(ii) $\hat{k}(\sigma)=0, \sigma \in \Sigma \backslash(P \times Q \times \bar{Q})$;

(iii) $0 \leqslant \hat{k}(\sigma) \leqslant I_{d_{\sigma}}$ for all $\sigma \in \Sigma$;

(iv) $\|k\|_{1} \leqslant(\nu(P \times \bar{Q}) / v(Q))^{\frac{1}{2}}$.

(Here, $P \times Q$ denotes the set $\mathrm{U}_{\sigma \in P, \eta \in Q} \sigma \times \eta$.)

Proof. Let $f=\sum_{\eta \in Q} d_{\eta} \chi_{\eta}, g=\sum_{\zeta \in P \times \bar{Q}} d_{\zeta} \chi_{\zeta}$, and

$$
k=\frac{1}{v(Q)} f \cdot g=\frac{1}{v(Q)} \sum_{\eta \in Q} \sum_{\zeta \in P \times \bar{Q}} d_{\eta} d_{\zeta}\left[\sum_{\zeta \in \eta \times \zeta} n_{\eta \zeta}(\epsilon) \chi_{\zeta}\right] .
$$


It is easily seen that

$$
\hat{k}(\sigma)=\left[\frac{1}{\nu(Q)} \sum_{\eta \in Q} \sum_{\zeta \in P \times \bar{Q}} \frac{d_{\eta} \cdot d_{\zeta}}{d_{\sigma}} n_{\eta \zeta}(\sigma)\right] I_{d_{\sigma}} .
$$

Notice that $n_{\eta \zeta}(\sigma)=n_{\sigma \bar{\eta}}(\zeta)$, and for $\alpha \in P, \eta \in Q, \sum_{\zeta \in P \times \bar{Q}} d_{\zeta} n_{\sigma \bar{\eta}}(\zeta)=d_{\sigma} d_{\eta}$. Hence, (i) follows from (1). (iii) also follows from (1) because for any $\sigma \in \Sigma$,

$$
\sum_{\zeta \in P \times \bar{Q}} d_{\zeta} d_{\sigma \bar{\eta}}(\zeta) \leqslant \sum_{\zeta \in \Sigma} d_{\zeta} n_{\sigma \bar{\eta}}(\zeta)=d_{\sigma} d_{\eta}
$$

(ii) results from (1) together with the fact that for $\sigma \notin P \times Q \times \bar{Q}$, and for any $\eta \in Q, \zeta \in P \times Q, n_{\eta \zeta}(\sigma)=0$. Finally,

$$
\|k\|_{1} \leqslant \frac{1}{\nu(Q)}\|f\|_{2}\|g\|_{2}=\left[\frac{\nu(P \times \bar{Q})}{\nu(Q)}\right]^{\frac{1}{2}} .
$$

(6.3) Definition. Let $M \in \mathbf{R}, M \geqslant 1$. A family $\Omega$ of finite non-empty subsets of $\Sigma$ is called a test family of order $M$ if $\sup \{v(P \times P \times \bar{P}) / \nu(P) \mid P \in \Omega\}<\infty$.

This is a generalization of the definition given by Edwards and Ross (1974).

(6.4) THEOREM. Let $\Omega$ be a test family of order $M$, and suppose that $R \subseteq \Sigma$ is of type central $\Lambda(r, s)$ for some $r, s \in[1, \infty)$ with constant $\kappa$. Then for any $P \in \Omega$,

$$
\nu(R \cap P) \leqslant \kappa^{s^{\prime}} M^{\left(s^{\prime} / 2\right)}[v(P)]^{s^{\prime}} / r .
$$

Proof. Choose a function $k \in Z T(G)$ satisfying (6.2) (i)-(iv), with $P=Q$. Let $g=\Sigma_{\sigma \in R \cap P} d_{\sigma} \chi_{\sigma}$. It is clear that

$$
v(R \cap P)=g * k(e) \leqslant\|k\|_{r}\|g\|_{r} \leqslant \kappa\|k\|_{r}\|\hat{g}\|_{s} .
$$

Now $\|\hat{g}\|_{s}=\left(\sum_{\sigma \in R \cap P} d_{\sigma}^{2}\right)^{1 / s}=\nu(R \cap P)^{1 / s}$. Thus $\nu(R \cap P)^{1 / s^{\prime}} \leqslant \kappa\|k\|_{r}$. But by Hölder's inequality,

$$
\begin{gathered}
\|k\|_{r^{\prime}} \leqslant\|k\|_{1}^{1-2 / r}\|k\|_{2}^{2 / r} \leqslant[\nu(P \times \bar{P}) / \nu(P)]^{\frac{1}{2}-1 / r}[\nu(P \times P \times \bar{P})]^{1 / r} \\
\leqslant M^{\frac{1}{2}}[\nu(P)]^{1 / r}
\end{gathered}
$$

since $\nu(P \times \bar{P}) \leqslant \nu(P \times P \times \bar{P}))$. This completes the proof.

Theorem (6.4) combines with (5.1) (ii) to prove:

(6.5) Corollary. Let $\Omega$ be a test family of order $M$. If $R$ is a p-Sidon set then there is $\kappa \in \mathbf{R}$ such that for every $P \in \Omega$ with $\nu(P) \geqslant 3$,

$$
\nu(R \cap P) \leqslant \kappa M^{p /(2-p)}[\log \nu(P)]^{p /(2-p)} .
$$

(6.6) ExAmple. Recall that a subset $P$ of $\Sigma$ is called a subhypergroup if $\bar{P} \subseteq P$ and $P \times P \subseteq P$. Let $\Omega$ be the family of all finite subhypergroups of $\Sigma$. Then $\Omega$ is a test family of order 1 . 
(6.7) Notation. Let $\sigma \in \Sigma$. Denote by $\sigma^{n}$ the subset $\sigma \times \ldots \times \sigma(n$ times $)$ of $\Sigma$. Let

$$
S_{r}(\sigma)=\bigcup_{\substack{j, k \geqslant 0 \\ j+k=r}} \sigma^{j} \times \bar{\sigma}^{k}, \quad S_{0}(\sigma)=\{1\}
$$

the trivial representation, and let $P_{n}(\sigma)=\bigcup_{r=0}^{n} S_{r}$. Notice that $P_{n}(\sigma)$ contains $\bigcup_{r=0}^{n} \sigma^{r}$. The latter set is denoted $A(\sigma, n)$ by Benke (1972). The two sets are equal if $\sigma=\bar{\sigma}$. If $G$ is abelian, $P_{n}(\sigma)$ is the arithmetic progression of length $n$ generated by $\sigma$.

(6.8) Lemma. For each $\sigma \in \Sigma$, there is a polynomial $p$ with degree at most $2 d_{\sigma}^{2}$ such that, for $n$ sufficiently large, $\nu\left(P_{n}(\sigma)\right)=p(n)$. If $\sigma=\bar{\sigma}$, the degree of $p$ is at $\operatorname{most} d_{\sigma^{2}}^{2}$

Proof. Let $J_{\sigma} \subseteq L^{2}(G)$ be the two-sided convolution ideal generated by $\left(1+\chi_{\sigma}+\bar{\chi}_{\sigma}\right)$. It is not hard to see that $J_{\sigma}$ is the linear span of the function 1 together with the coordinate functions $\sigma_{i_{j}}, \bar{\sigma}_{i j}, i, j=1, \ldots, d_{\sigma^{*}}$. Thus $\operatorname{dim} J_{\sigma} \leqslant 2 d_{\sigma}^{2}+1$ (if $\left.\sigma=\bar{\sigma}, \operatorname{dim} J_{\sigma}=d_{\sigma}^{2}+1\right)$.

Let $J_{\sigma}^{n}$ be the subspace of $L^{2}$ spanned by functions of the form $\phi_{1} \cdot \ldots \cdot \phi_{n}$, $\phi_{j} \in J_{\sigma}$. Notice that $J_{\sigma}^{n}$ is spanned by co-ordinate functions of the representations 1 and $\sigma^{j} \otimes \bar{\sigma}^{k}, 1 \leqslant j+k \leqslant n$ and hence $\operatorname{dim} J_{\sigma}^{n}=\sum_{\zeta \in P_{n}(\sigma)} d_{\zeta}^{2}=\nu\left(P_{n}(\sigma)\right) . J_{\sigma}^{n}$ as a vector space is isomorphic to the vector space of polynomials of degree $n$ in $\operatorname{dim} J_{\sigma}$ variables. Thus by Lemma 8.9 of Mayer (1967), there exists a polynomial $p$ of degree $\operatorname{dim}\left(J_{\sigma}-1\right)$ such that $\operatorname{dim} J_{\sigma}^{n}=p(n)$.

(6.9) Propositions. $\left\{P_{n}(\sigma) \mid n \in \mathrm{N}\right\}$ is a test family.

Proof. Now $\overline{P_{n}(\sigma)}=P_{n}(\sigma)$, and one has $P_{n}(\sigma) \times P_{n}(\sigma) \times P_{n}(\sigma)=P_{3 n}(\sigma)$. By (6.8), for $n$ sufficiently large,

$$
\frac{\nu\left(P_{3 n}(\sigma)\right)}{\nu\left(P_{n}(\sigma)\right)} \leqslant 3^{2 d_{\sigma}^{2}}+\frac{1}{2} .
$$

(6.10) Remarks. Applying (6.4) to (6.9) gives:

Let $\sigma \in \Sigma$, and suppose that $R \subseteq \Sigma$ is of type central $\Lambda(r, s), r, s \in[1, \infty)$. Then there exists $\kappa \in \mathbf{R}$ such that $\nu\left(R \cap P_{n}(\sigma)\right) \leqslant \kappa n^{2 s^{*} d^{2} / p}$ for all $n$; if $\sigma=\bar{\sigma}$,

$$
\nu\left(R \cap P_{n}(\sigma)\right) \leqslant \kappa n^{s^{\prime} d_{\sigma^{2}} / p} .
$$

In particular, if $R$ is a central $\Lambda(p)$ set $(p>2), \nu\left(R \cap P_{n}(\sigma)\right) \leqslant \kappa n^{4 d_{\sigma} / p}$ for all $n$. Benke (1972) shows that if $R$ is a central $\Lambda(p)$ set, $\operatorname{card}(R \cap A(\sigma, n)) \leqslant O\left(n^{4 d_{\sigma^{2}} / p}\right)$. The above is an improvement of his result, since $P_{n}(\sigma) \supseteq A(\sigma, n)$ and also, for any set $R, \nu(R) \geqslant$ card $R$, equality prevailing if and only if $d_{\sigma}=1$ for all $\sigma \in R$.

(6.11) Proposition. Let $G$ be an infinite compact group. Then $\Sigma$ has an infinite test family. 
Proof. If there is a $\sigma \in \Sigma$ such that $[\sigma]$ is infinite, then (6.9) provides such a test family. If not, for every $\sigma \in \Sigma,[\sigma]$ is finite. In this case, the family of finite subhypergroups of $\Sigma$ is an infinite test family.

Applying the above to (6.4) gives

(6.12) COROLlaRY. Let $G$ be an infinite compact group. Then $\Sigma$ is never of type central $\Lambda(p, q)$ for $p>q^{\prime}$.

(6.13) Proposition. $\Sigma(\mathscr{S} \mathscr{U}(2))$ contains no infinite sets of type central $\Lambda(p, q)$ for $p>\frac{3}{2} q^{\prime}, p, q \in[1, \infty[$.

Proof. Let $\sigma_{l}$ be the (unique) irreducible representation of $\mathscr{S} \mathscr{U}(2)$ of dimension $2 l+1, l \in\left\{0, \frac{1}{2}, 1, \ldots\right\}$ (Hewitt and Ross, 1972 (29.13)). Then for $n \in N$,

$$
P_{n}\left(\sigma_{\frac{1}{2}}\right)=\left\{\sigma_{0}, \sigma_{\frac{1}{2}}, \ldots, \sigma_{\frac{1}{2} n}\right\} .
$$

Suppose that $R=\left\{\sigma_{n_{i}} \mid 2 n_{i} \in \mathbf{N}\right\}$ is an infinite set of type $\Lambda(p, q)$. One has

$$
\nu\left(P_{n_{i}}\left(\sigma_{\frac{1}{2}}\right) \cap R\right)=\sum_{j=1}^{i}\left(2 n_{j}+1\right)^{2}
$$

and

$$
\nu\left(P_{n}\left(\sigma_{\frac{1}{2}}\right)\right)=\sum_{j=0}^{n}(2 j+1)^{2}=O\left(\frac{4 n^{3}}{3}\right) .
$$

Thus by (6.10) there is a constant $\kappa$ such that for all $i$,

$$
\left(2 n_{i}+1\right)^{2} \leqslant \nu\left(P_{n_{i}}\left(\sigma_{\frac{1}{i}}\right) \cap R\right) \leqslant \kappa\left(n_{i}^{3}\right)^{q / p} .
$$

Since $R$ is infinite, this implies that $p \leqslant \frac{3}{2} q^{\prime}$.

Hence, by (5.1)(ii), $\Sigma(\mathscr{S} \mathscr{U}(2))$ contain no infinite $p$-Sidon sets for any $p \in[1,2[$.

\section{REFERENCES}

G. Benke (1974), “On the hypergroup structure of central $\Lambda(p)$ sets", Pacific J. Math. 50, 19-27. M. Bożejko and T. Pytlik (1972), "Some types of lacunary Fourier series", Colloq. Math. 25, 117-124.

C. Cecchini (1972), "Lacunary series on Lie groups", J. Functional Anal. 11, 191-203.

R. E. Edwards and K. A. Ross (1974), "p-Sidon sets", J. Functional Anal. 15, 404-427.

A. Figà-Talamanca and D. Rider (1966), "A theorem on random Fourier series on noncommutative groups", Pacific J. Math. 16, 505-514.

L.-S. Hahn, "Fourier series with gaps", Preprint.

E. Hewitt and K. A. Ross (1972), Abstract Harmonic Analysis, Vol. II (Springer-Verlag, Berlin).

R. Mayer (1967), "Summation of Fourier series on compact groups", Amer; J. Math. 89, 661-692.

W. A. Parker (1972), "Central Sidon and central $\Lambda(p)$ sets", J. Austral. Math. Soc. 14, 62-74. J. F. Price (1971), "Non ci sono insiemi di tipo $\Lambda(p)$ per $\mathscr{S} \mathscr{U}(2)$ ", Boll. d'un Mat. Ital. (4) 4, 879-881. 
D. Rider (1972), "Central lacunary sets", Monatsh. Math. 76, 328-338.

W. Rudin (1962), Fourier Analysis on Groups (Wiley Interscience, New York).

Department of Mathematics

Institute of Advanced Studies

Australia National University

Canberra 